\title{
Looking past PD-L1: expression of immune checkpoint TIM-3 and its ligand galectin-9 in cervical and vulvar squamous neoplasia
}

\author{
Jacob Curley ${ }^{1} \cdot$ Mark R. Conaway ${ }^{2} \cdot$ Zachary Chinn $^{1} \cdot$ Linda Duska $^{3} \cdot$ Mark Stoler $^{1} \cdot$ Anne M. Mills $\mathbb{D}^{1}$
}

Received: 9 September 2019 / Revised: 26 November 2019 / Accepted: 28 November 2019 / Published online: 6 March 2020

(c) The Author(s), under exclusive licence to United States \& Canadian Academy of Pathology 2020

\begin{abstract}
Immunotherapies targeting the PD-1/PD-L1 pathway have shown some success in cervical and vulvar squamous cell carcinomas, but little is known about the potential vulnerability of these tumors to other checkpoint inhibitors. TIM-3 is a checkpoint molecule that exerts immunosuppressive function via its interaction with Gal-9. TIM-3 and Gal-9 have been identified on a variety of malignancies but have not been studied in cervical and vulvar cancers, nor has their relationship to PD-L1 been established. Sixty-three cervical and vulvar invasive $(n=34)$ and intraepithelial lesions $(n=29)$ were assessed for TIM-3, Gal-9, and PD-L1 in tumor/lesional cells and associated immune cells. Tumoral TIM-3 expression was identified in $85 \%$ of squamous cell carcinomas but only $21 \%$ of intraepithelial lesions $(p<0.0001)$. When immune cells were also accounted for, $97 \%$ of invasive and $41 \%$ of intraepithelial lesions had a TIM-3 combined positive score (CPS) $\geq 1(p<$ 0.0001). Tumoral membranous expression of Gal-9 was seen in $82 \%$ of squamous cell carcinomas and $31 \%$ of intraepithelial lesions ( $p=0.0001$ ); nearly all cases had Gal-9-positive immune cells. Tumoral PD-L1 was seen in $71 \%$ of squamous cell carcinomas and $10 \%$ of intraepithelial lesions $(p<0.0001)$, while the PD-L1 CPS was $\geq 1$ in 82 and $21 \%$, respectively $(p<$ 0.0001). There were no significant differences in TIM-3, GAL-9, or PD-L1 expression in cervical vs. vulvar neoplasms, nor was HPV status significantly associated with any of the three markers. Dual TIM-3/Gal-9 expression was present in the majority (86\%) of PD-L1-positive cases including 100\% of PD-L1-positive squamous cell carcinomas, suggesting a possible role for TIM-3 checkpoint inhibition in concert with anti-PD-1/PD-L1.
\end{abstract}

\section{Introduction}

In recent years, drugs targeting the PD-1/PD-L1 immune checkpoint pathway have shown promise in a variety of tumor types, including cervical squamous cell carcinomas [1-4]. Multiple clinical trials investigating anti-PD-1/PD-L1

Supplementary information The online version of this article (https:// doi.org/10.1038/s41379-019-0433-3) contains supplementary material, which is available to authorized users.

Anne M. Mills

AnneMills1@gmail.com

1 Department of Pathology, University of Virginia, Charlottesville, VA, USA

2 Department of Public Health Sciences, Division of Translational Research and Applied Statistics, University of Virginia, Charlottesville, VA, USA

3 Departments of Obstetrics and Gynecology, Division of Gynecologic Oncology, University of Virginia, Charlottesville, VA, USA checkpoint inhibitors in cervical squamous cell carcinomas are ongoing, and primarily focus on the use of these drugs in combination with conventional chemotherapy in advanced stage and refractory tumors (clinicaltrials. gov: NCT03808857; NCT03912402; NCT03104699; NCT02257528; NCT03635567). Moreover, recent success of the PD-1 inhibitor pembrolizumab in a subset of PD-L1positive recurrent/refractory cervical squamous cell carcinomas has led to the Food \& Drug Administration approval of the drug in this context [5]. While no studies have focused specifically on vulvar squamous cell carcinoma, this tumor type is included in some trials investigating the use of anti-PD-1 checkpoint inhibitors in rare malignancies (NCT02834013; NCT02834013) and one case report has demonstrated an impressive response of a vulvar squamous cancer to pembrolizumab [6].

However, as has been shown in other tumor types, early evidence suggests that an anti-PD-1/PD-L1 immunotherapy response is far from guaranteed for gynecologic squamous cancers, and the majority of patients do not show sustained benefit $[4,7]$. 
The ultimate failure of single-agent immunotherapy in many patients may be attributable to the presence of additional mechanisms of immune evasion [8, 9]. One such mechanism is the immunosuppressive checkpoint molecule T-cell immunoglobulin and mucin-domain containing-3 (TIM-3). TIM-3 is normally expressed on the surface of fully differentiated $\mathrm{T}_{\mathrm{H}} 1$ cells and is activated by binding to its ligand galectin-9 (Gal-9), resulting in a downregulation of cytotoxic $\mathrm{T}$ cells activity and increase in regulatory $\mathrm{T}$-cell activity [10, 11]. Normally this receptor-ligand binding pair plays a role in modulating alloimmune response and promoting immune tolerance, however it may also be co-opted by malignancy. TIM-3 expression has been demonstrated within the immune microenvironment of a wide array of neoplasms including melanoma, gastric carcinoma, urothelial carcinoma, renal cell carcinoma, and prostatic carcinoma [12-15], and has also been identified on both tumor and tumor-associated immune cells in endometrial carcinoma [16]. Several early clinical trials of monoclonal antibodies against TIM-3 are underway in advanced solid malignancies, and include patients with cervical carcinoma (clinicaltrials.gov: NCT03489343, NCT02817633, NCT03680508, NCT02608268, NCT03652077). Expression of the TIM-3 ligand Gal-9 has also been demonstrated in a variety of epithelial tumors including hepatocellular carcinoma, gastric carcinoma, and breast carcinoma [17-20]. In addition to its immunomodulatory role, Gal-9 has been shown to contribute to tumor cell aggregation and adhesion [19, 21].

The relationship between TIM-3 and GAL-9 has not been previously investigated in cervical and vulvar malignancies and precursor lesions, nor have these markers been assessed in concert with PD-L1. We therefore evaluated TIM-3, GAL-9, and PD-L1 expression in cervical and vulvar squamous intraepithelial lesions and carcinomas to assess the possible biologic basis for TIM-3 checkpoint inhibition, potentially in combination with anti-PD-1/PD$\mathrm{L} 1$, in these neoplasms.

\section{Materials and methods}

\section{Case selection}

This retrospective study was approved by the institutional review board of the University of Virginia. Whole sections of formalin-fixed, paraffin-embedded tissue from 63 cases of cervical and vulvar neoplasia were collected from the archival pathology files and reviewed. Consecutive cases were selected based on a natural language search; only those cases with sufficient tumor available for additional studies were included. The cases were comprised of cervical squamous cell carcinoma ( $n=15)$, HPV-associated vulvar squamous cell carcinoma $(n=16)$, differentiated vulvar intraepithelial neoplasia (dVIN)-associated vulvar squamous cell carcinoma $(n=3), \mathrm{dVIN}(n=2)$, VIN $3(n=13)$, and CIN $3(n=14)$. The intraepithelial lesions were not matched to the invasive carcinomas; rather, they derived from independent patients. All cases were reviewed to confirm histologic typing and invasive vs. in situ status, and all vulvar cases had p16 (CINTEC Plus Histology kit Roche/Ventana, dilution of 1:2) to confirm HPV-associated vs. unassociated status. For invasive carcinomas, strong diffuse staining in $\geq 70 \%$ of tumor cells was considered consistent with HPV association, while in intraepithelial lesions block-like staining extending beyond the lower epithelial third was required.

\section{TIM-3, Gal-9, and PD-L1 immunohistochemistry}

All immunohistochemical staining was performed using whole sections. Immunohistochemical staining for TIM-3 (clone ab185703, Abcam; dilution 1:400;) and Gal-9 (Clone ab153673, Abcam; dilution 1:100) was performed using the Ventana Benchmark Ultra in the University of Virginia Biorepository and Tissue Research Facility. PD-L1 (Spring Biosciences, SP142, dilution 1:200) immunostaining was performed on a Leica Bond III. PD-L1 immunostaining platform selection was based on the fact that this antibody has been internally validated on this platform against the Dako 22C3 antibody with comparable performance in both tumor cells and immune cells, whereas experience in our laboratory and elsewhere suggests that the SP142 antibody has less sensitivity for tumor cell expression when the Ventana instrument is used [22, 23]. Tonsillar tissue was used as the control for TIM-3 and Gal-9, while placental tissue was used for PD-L1.

Immunostaining was scored manually at the microscope by two independent reviewers (JDC and AM). All three stains were scored both using the Tumor Proportion Score (TPS), which accounts for membranous tumoral staining alone, and the combined positive score (CPS), which considers both membranous tumoral staining and associated mononuclear immune cell (lymphocyte and macrophage) staining. The rationale for using both scoring systems is that the most clinically relevant methodology to assess TIM-3 and Gal-9 remains unknown, therefore providing data using both systems seems prudent for this early descriptive analysis. PD-L1 expression using the TPS and/or the CPS has proven significant in different clinical settings and with the ideal scoring system varying based on tumor type and anatomic location. Importantly, the CPS is the approved scoring system for the use of pembrolizumab in recurrent/ advanced cervical cancer [5].

For both scores, tumoral staining interpretation was based exclusively on membranous expression due to the fact that cell surface localization is required to facilitate engagement of the immune checkpoint axis for each of these molecules. 
Table 1 TIM-3 and Gal-9 expression

\begin{tabular}{|c|c|c|c|c|c|}
\hline & $\begin{array}{l}\text { Tumoral } \\
\text { TIM-3 } \geq 1 \%\end{array}$ & $\begin{array}{l}\text { TIM-3 } \\
\text { CPS } \geq 1\end{array}$ & $\begin{array}{l}\text { Tumoral } \\
\text { Gal- } 9 \geq 1 \%\end{array}$ & $\begin{array}{l}\text { Gal-9 } \\
\text { CPS } \geq 1\end{array}$ & $\begin{array}{l}\text { TIM-3 and Gal-9 } \\
\text { Co-expression }(\mathrm{CPS} \geq 1)\end{array}$ \\
\hline $\begin{array}{l}\text { All Intraepithelial } \\
\text { Lesions }(\boldsymbol{n}=29)\end{array}$ & $\begin{array}{l}\text { 21\% }(6 / 29) \\
\text { Staining } \\
\text { extent: } \\
1-5 \%: 6\end{array}$ & $\begin{array}{l}\text { 41\% (12/29) } \\
\text { CPS value: } \\
1-5: 9 \\
6-10: 2 \\
11-25: 1\end{array}$ & $\begin{array}{l}\text { 31\% }(9 / 29) \\
\text { Staining extent: } \\
1-5 \%: 6 \\
6-10 \%: 1 \\
11-25 \%: 1 \\
25-50 \%: 1\end{array}$ & $\begin{array}{l}\text { 100\% }(29 / \\
\text { 29) } C P S \\
\text { value: } \\
\text { 1-5: } 9 \\
6-10: 7 \\
\text { 11-25: } 8 \\
\text { 26-50: } 4 \\
>50: 1\end{array}$ & $41 \%(12 / 29)$ \\
\hline CIN $3(n=14)$ & $\begin{array}{l}\text { 21\% (3/14) } \\
\text { Staining } \\
\text { extent: } \\
1-5 \%: 3\end{array}$ & $\begin{array}{l}\text { 64\% }(9 / 14) \\
\text { CPS value: } \\
\text { 1-5: } 6 \\
\text { 6-10: } 2 \\
\text { 11-25: } 1\end{array}$ & $\begin{array}{l}\text { 43\% }(6 / 14) \\
\text { Staining extent: } \\
1-5 \%: 3 \\
6-10 \%: 1 \\
11-25 \%: 1 \\
25-50 \%: 1\end{array}$ & $\begin{array}{l}\text { 100\% } \\
(14 / 14) \text { CPS } \\
\text { value: } \\
1-5: 9 \\
6-10: 2 \\
11-25: 3\end{array}$ & $\mathbf{6 4 \%}(9 / 14)$ \\
\hline $\operatorname{VIN} 3(n=13)$ & $\begin{array}{l}\mathbf{2 3 \%}(3 / 13) \\
\text { Staining } \\
\text { extent: } \\
1-5 \%: 3\end{array}$ & $\begin{array}{l}\mathbf{2 3 \%}(3 / 13) \\
\text { CPS value: } \\
1-5: 3\end{array}$ & $\begin{array}{l}\mathbf{2 3 \%}(3 / 13) \\
\text { Staining extent: } \\
1-5 \%: 3\end{array}$ & $\begin{array}{l}\text { 100\% } \\
\text { (13/13) CPS } \\
\text { value: } \\
6-10: 3 \\
11-25: 5 \\
26-50: 4 \\
>50: 1\end{array}$ & $23 \%(3 / 13)$ \\
\hline$d V I N(n=2)$ & 0\% $(0 / 2)$ & 0\% $(0 / 2)$ & 0\% $(0 / 2)$ & $\begin{array}{l}\text { 100\% }(2 / 2) \\
\text { CPS value: } \\
6-10: 2\end{array}$ & 0\% $(0 / 2)$ \\
\hline All Invasive SCC $(\boldsymbol{n}=34)$ & $\begin{array}{l}\mathbf{8 5 \%}(29 / 34) \\
\text { Staining } \\
\text { extent: } \\
1-5 \%: 10 \\
6-10 \%: 6 \\
11-25 \%: 5 \\
25-50 \%: 5 \\
>50 \%: 3\end{array}$ & $\begin{array}{l}97 \% \text { (33 of } \\
34) \text { CPS } \\
\text { value: } \\
1-5: 14 \\
6-10: 4 \\
11-25: 4 \\
25: 50: 4 \\
>50: 7\end{array}$ & $\begin{array}{l}\mathbf{8 2 \%}(28 / 34) \\
\text { Staining extent: } \\
\text { 1-5\%: } 15 \\
6-10 \%: 9 \\
11-25 \%: 2 \\
25-50 \%: 2\end{array}$ & $\begin{array}{l}\mathbf{1 0 0 \%} \\
(34 / 34) \text { CPS } \\
\text { value: } \\
1-5: 2 \\
6-10: 8 \\
11-25: 6 \\
26-50: 13 \\
>50: 5\end{array}$ & 97\% (33/34) \\
\hline Cervical SCC $(n=15)$ & $\begin{array}{l}\text { 87\% (13/15) } \\
\text { Staining } \\
\text { extent: } \\
1-5 \%: 3 \\
6-10 \%: 3 \\
11-25 \%: 0 \\
25-50 \%: 4 \\
>50 \%: 3\end{array}$ & $\begin{array}{l}\mathbf{1 0 0 \%} \\
\text { (15/15) CPS } \\
\text { value: } \\
1-5: 3 \\
6-10: 2 \\
11-25: 1 \\
25: 50: 4 \\
>50: 5\end{array}$ & $\begin{array}{l}\text { 80\% }(12 / 15) \\
\text { Staining extent: } \\
1-5 \%: 5 \\
6-10 \%: 4 \\
11-25 \%: 2 \\
25-50 \%: 1\end{array}$ & $\begin{array}{l}\mathbf{1 0 0 \%} \\
\text { (15/15) CPS } \\
\text { value: } \\
1-5: 1 \\
6-10: 2 \\
11-25: 2 \\
26-50: 6 \\
>50: 4\end{array}$ & $100 \%(15 / 15)$ \\
\hline $\begin{array}{l}\text { Vulvar SCC, HPV-Associated } \\
(n=16)\end{array}$ & $\begin{array}{l}\text { 81\% (13/16) } \\
\text { Staining } \\
\text { extent: } \\
1-5 \%: 5 \\
6-10 \%: 3 \\
11-25 \%: 4 \\
25-50 \%: 1\end{array}$ & $\begin{array}{l}\text { 94\% (15/16) } \\
\text { CPS value: } \\
1-5: 9 \\
6-10: 2 \\
11-25: 2 \\
>50: 2\end{array}$ & $\begin{array}{l}\mathbf{7 5 \%}(12 / 16) \\
\text { Staining extent: } \\
1-5 \%: 6 \\
6-10 \%: 5 \\
11-25 \%: 0 \\
25-50 \%: 1\end{array}$ & $\begin{array}{l}\text { 100\% } \\
\text { (16/16) CPS } \\
\text { value: } \\
1-5: 1 \\
6-10: 4 \\
11-25: 4 \\
26-50: 6 \\
>50: 1\end{array}$ & $94 \%(15 / 16)$ \\
\hline $\begin{array}{l}\text { Vulvar SCC, dVIN- } \\
\text { associated }(n=3)\end{array}$ & $\begin{array}{l}\text { 100\% (3/3) } \\
\text { Staining } \\
\text { extent: } \\
1-5 \%: 2 \\
6-10 \%: 0 \\
11-25 \%: 1\end{array}$ & $\begin{array}{l}100 \%(3 / 3) \\
\text { CPS value: } \\
1-5: 2 \\
11-25: 1\end{array}$ & 0\% $(0 / 3)$ & $\begin{array}{l}\mathbf{1 0 0 \%}(3 / 3) \\
\text { CPS value: } \\
\text { 6-10: } 2 \\
\text { 26-50: } 1\end{array}$ & $100 \%(3 / 3)$ \\
\hline
\end{tabular}

The proportion of positive cases (for tumoral staining $\geq 1 \%$; for $\mathrm{CSP} \geq 1$ ) is indicated in bold. The number of cases falling into different expression ranges (either by $\%$ tumoral staining or by CPS value) is cascaded beneath for individual TIM-3 and Gal-9 expression columns. Co-expression is based on the CPS $\geq 1$ threshold for both markers

Macrophage staining was also scored based on membranous expression, however lymphocyte scoring was based on membranous/cytoplasmic expression because the high nuclear:cytoplasm ratio of lymphocytes typically precludes acute distinction of membranous vs. cytoplasmic staining therefore expression in both compartments is considered positive in existing PD-L1 scoring schema.

The TPS was assigned based on the percentage of viable tumor showing partial or complete membranous staining of any intensity. Tumoral/lesional expression was scored on a continuous basis from 0 to $100 \%$ and considered positive in the context of $\geq 1 \%$ staining.

The CPS was calculated as follows: CPS $=[$ (number of positive tumor cells, lymphocytes, and macrophages)/ (total number of tumor cells) $\times 100$; the maximum allowable CPS is 100 [24]. CPS was assigned on a continuous basis from 0 to 100 and a score of $\geq 1$ was considered positive. 


\section{Tumor-infiltrating lymphocytes (TILs)}

TILs were assessed according to the criteria proposed by Salgado et al. to produce a TIL stromal score (TIL-SS) [25]. According to these recommendations, mononuclear inflammatory cells were assessed at the tumoral-stromal interface and scored based on the percentage of stroma occupied by these inflammatory cells using the area of stromal tissue (rather than number of stromal cells) as the denominator. The assessment was averaged across the lesion represented in an entire tissue section, rather than based on a single "hot spot".

\section{Statistics}

The PD-L1 and TIM-3 scores were analyzed both as dichotomous, classifying scores as either 0 or greater than 0 , or as continuous variables. Statistical analysis for the binary outcomes was performed using the two-tailed Fischer Exact Probability Test (vassarstats.net). Scatterplots were used to describe the pattern of continuous PD-L1 and TIM-3 scores across groups defined by cervical or vulvar squamous intraepithelial lesions and carcinomas. Rank correlations and multivariate regression were used to describe the association between continuous PD-L1 and TIM-3 scores and TIL stroma scores. The nonparametric Kruskal-Wallis test was used to compare the TIL stroma scores across groups. Statistical analyses were performed by MRC.

\section{Results}

\section{TIM-3 expression}

TIM-3 was expressed in scattered lymphoid cells from lymphoid control tissue but was entirely negative in normal squamous epithelium (Supplemental figure). Across cervical and vulvar squamous neoplasms, membranous tumoral TIM-3 expression $\geq 1 \%$ was significantly more common among invasive vs. intraepithelial lesions (85\% vs. $21 \%, p<0.0001$ ) (Table 1, Figs. 1-3). Most TIM-3positive neoplasms had only focal epithelial staining, however three cases-all cervical squamous cell carcinomas-showed membranous TIM- 3 expression in $\geq 50 \%$ of cells. Immune cell TIM-3 expression was also significantly more common among squamous cell carcinomas when compared with intraepithelial lesions (85\% vs. $28 \%, p<0.0001$ ) (Table 1). The average CPS was 2 for intraepithelial lesions (range: $0-25$ ) and 22 for invasive

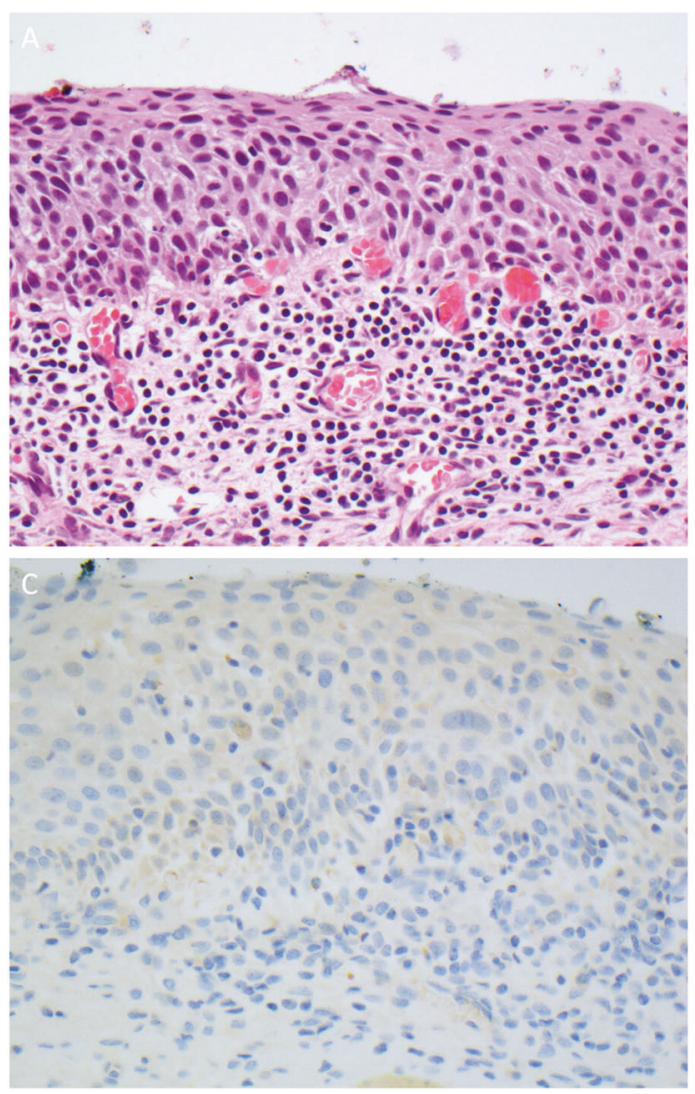

Fig. 1 PD-L1, TIM-3, and Gal-9 expression in squamous intraepithelial lesions. Intraepithelial lesions, such as the CIN 3 depicted in a, tended to have PD-L1 (b), and TIM-3 (c) expression limited to

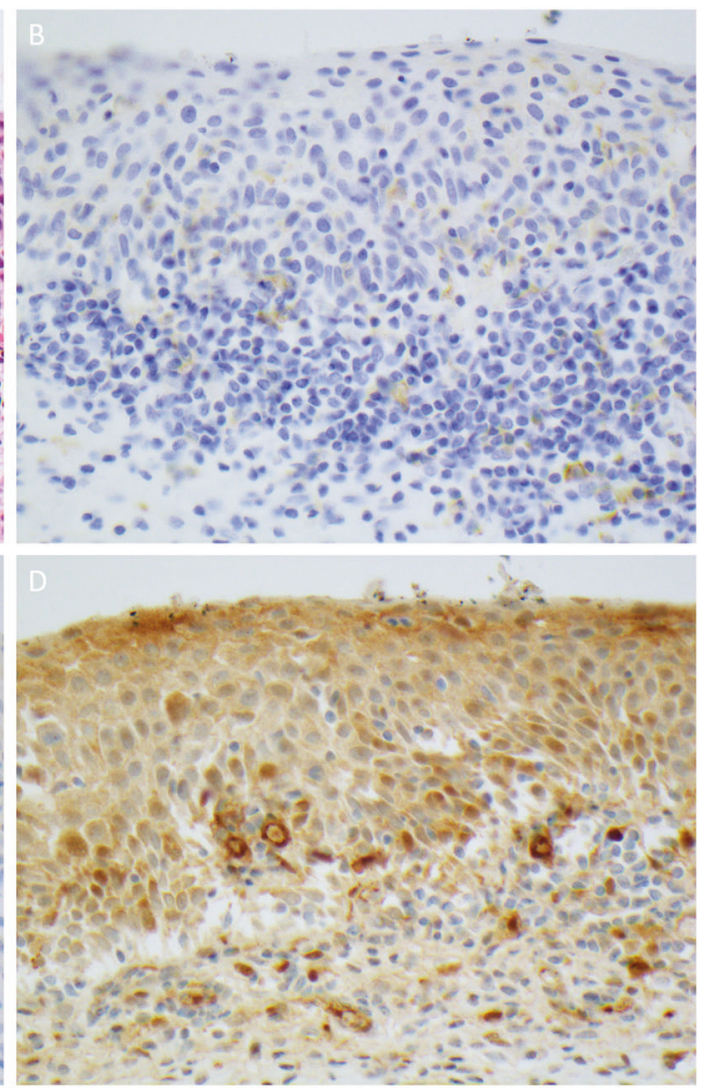

lymphocytes, whereas GAL-9 was typically positive in epithelial and stromal cells in a nuclear and cytoplasmic distribution. 
lesions (range: 0-100); 97\% of squamous cell carcinomas had a TIM-3 CPS $\geq 1$, whereas only $41 \%$ of intraepithelial lesions met this threshold $(p<0.0001)$. There was no significant difference in TIM-3 expression based on location (cervical vs. vulvar) for HPV-associated invasive tumors. However, significantly more cases of CIN 3 met the TIM- 3 CPS threshold of $\geq 1$ when compared with cases of VIN $3(64 \%$ vs. $23 \%, p=0.05)$. Although there were no significant differences identified in expression in HPVpositive vs. HPV-negative vulvar neoplasms, statistical power was limited by low numbers of HPV-negative invasive and intraepithelial neoplasia.

\section{Gal-9 expression}

Nuclear $+/$ - cytoplasmic expression of Gal-9 was identified across normal tissues, including lymphoid control tissue, cervical and vulvar stroma, and non-neoplastic cervical and vulvar squamous mucosa (Supplemental figure). In addition to the normal nuclear Gal-9 expression seen across epithelial tissues, a subset of invasive and intraepithelial lesions showed membranous expression
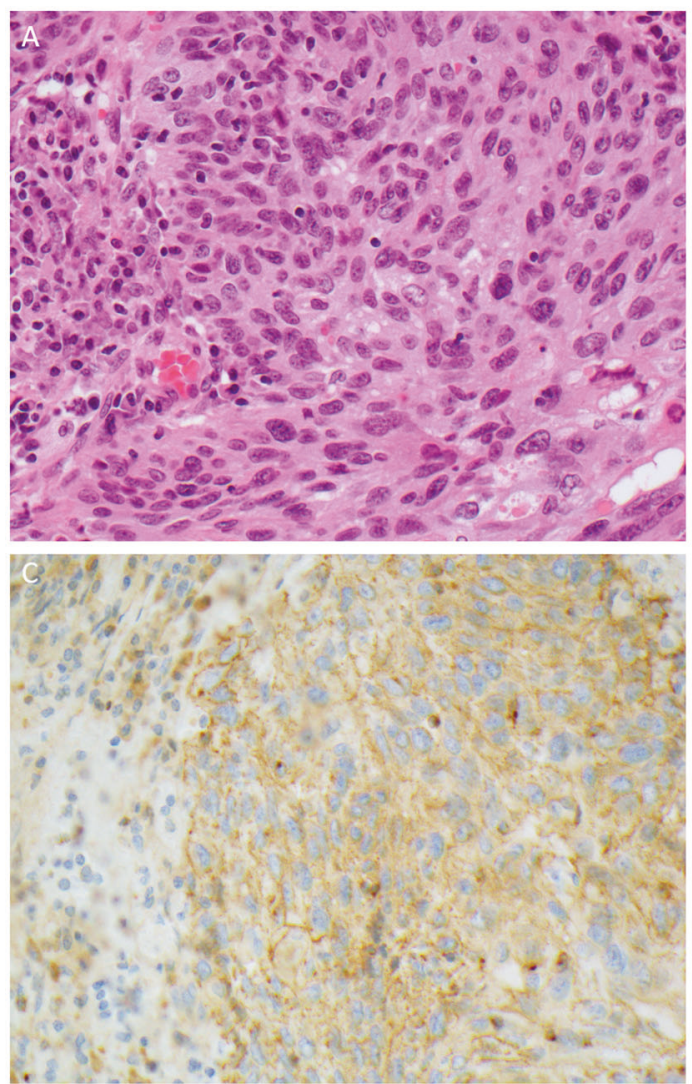

Fig. 2 PD-L1, TIM-3, and Gal-9 expression in cervical squamous cell carcinomas. The majority of cervical squamous cell carcinomas expressed PD-L1, with the highest expressers including the case illustrated in a which showed strong membranous epithelial staining as well as positivity in tumor-associated lymphocytes (b). TIM-3 (c) was consistent with cell-surface localization (Figs. 1-3). This membranous expression was often accompanied by a concomitant decrease in nuclear staining. Membranous tumoral expression $\geq 1 \%$ of Gal-9 was more common among squamous cell carcinomas vs. intraepithelial lesions $(82 \%$ vs. $31 \%, p=0.0001$ ) (Table 1). Membranous Gal-9 expression was often accompanied by a decrease or loss of nuclear/cytoplasmic expression in invasive cases. Gal-9-positive immune cells were identified in all but one case, and due to the near ubiquity of Gal-9-positive immune cells, all cases met the Gal-9 CPS threshold of $\geq 1$. The mean Gal-9 CPS was 17 (range: 1-60) for intraepithelial lesions and 31.2 (range: 5-100) for invasive carcinomas. There were no significant differences in Gal-9 expression based on tumor location or HPV status, although only a few HPV-negative cases were evaluated.

\section{PD-L1 expression}

PD-L1 expression was identified on villi in placental control tissue but was entirely negative in normal squamous control
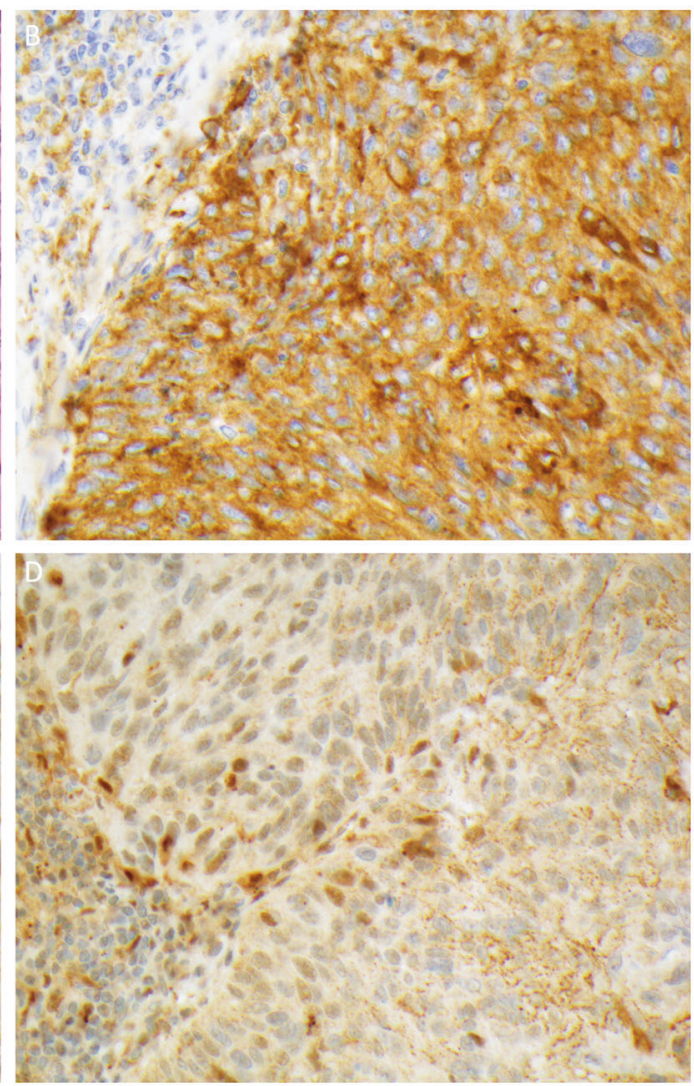

also positive in this case on both tumor cells and affiliated immune cells. Gal-9 (d) showed a different pattern than was seen in normal epithelium and intraepithelial lesions, with decreased nuclear and cytoplasmic staining and more prominent membranous expression in tumor cells as well as immune cell staining. 
tissue (Supplemental figure). PD-L1 expression was significantly more common among invasive squamous cell carcinomas vs. intraepithelial lesions using both tumoral staining $\geq 1 \%$ ( $71 \%$ vs. $10 \%, p<0.0001)$ and a CPS $\geq 1$ ( $82 \%$ vs. $21 \%, p<0.0001$ ) (Figs. $1-3$, Table 2 ). The mean CPS was 4.2 (range: 0-40) for intraepithelial lesions and 49.2 (range 0-100) for invasive carcinomas. PD-L1 expression did not differ significantly between cervical and vulvar HPV-associated cases. There were no significant differences among HPV-positive vs. HPV-negative vulvar cases, although statistical power was limited by the small number of HPV-negative cases assessed. PD-L1 staining results from these cases were previously reported in a study by Chinn et al. [26]

\section{TIM-3, Gal-9, and PD-L1 co-expression}

Using the CPS system, TIM-3/Gal-9 co-expression was seen in 97\% (33/35) of cases (Table 1). All 28 PD-L1positive squamous cell carcinomas $(82 \%, 28 / 34$ of total squamous cell carcinomas) co-expressed TIM-3 and Gal-9 at the CPS $\geq 1$ threshold, with a subset of cases showing high-level dual expression of both targetable checkpoint molecules. (Table 2, Fig. 4) In contrast, 15\% (5/34) of squamous cell carcinomas were TIM-3 and Gal-9-positive but negative for PD-L1 in both tumor and immune cells.

Overall, $41 \%$ (12/29) of intraepithelial lesions showed dual TIM-3/Gal-9 expression using the CPS expression $\geq 1$ cut-off. Simultaneous expression of TIM-3, Gal-9, and PD-L1 was highly uncommon among intraepithelial lesions $(7 \%, 2 / 29)$ while TIM-3 and Gal-9 co-expression in the absence of PD-L1 expression was seen in approximately one third (34\%, 10/29) of the intraepithelial lesions (Table 3).

\section{Tumor-infiltrating lymphocytes (TILs)}

The proportion of tumor-adjacent stroma occupied by inflammatory cells had a mean of $16.5 \%$ (range: $5-35 \%$ ) for intraepithelial lesions and $42 \%$ (range: 5-85\%) for invasive lesions. (Supplemental Table) On average, cervical squamous cell carcinomas showed higher levels of associated inflammation (mean: 52\%, range: $35-85 \%$ ) than vulvar
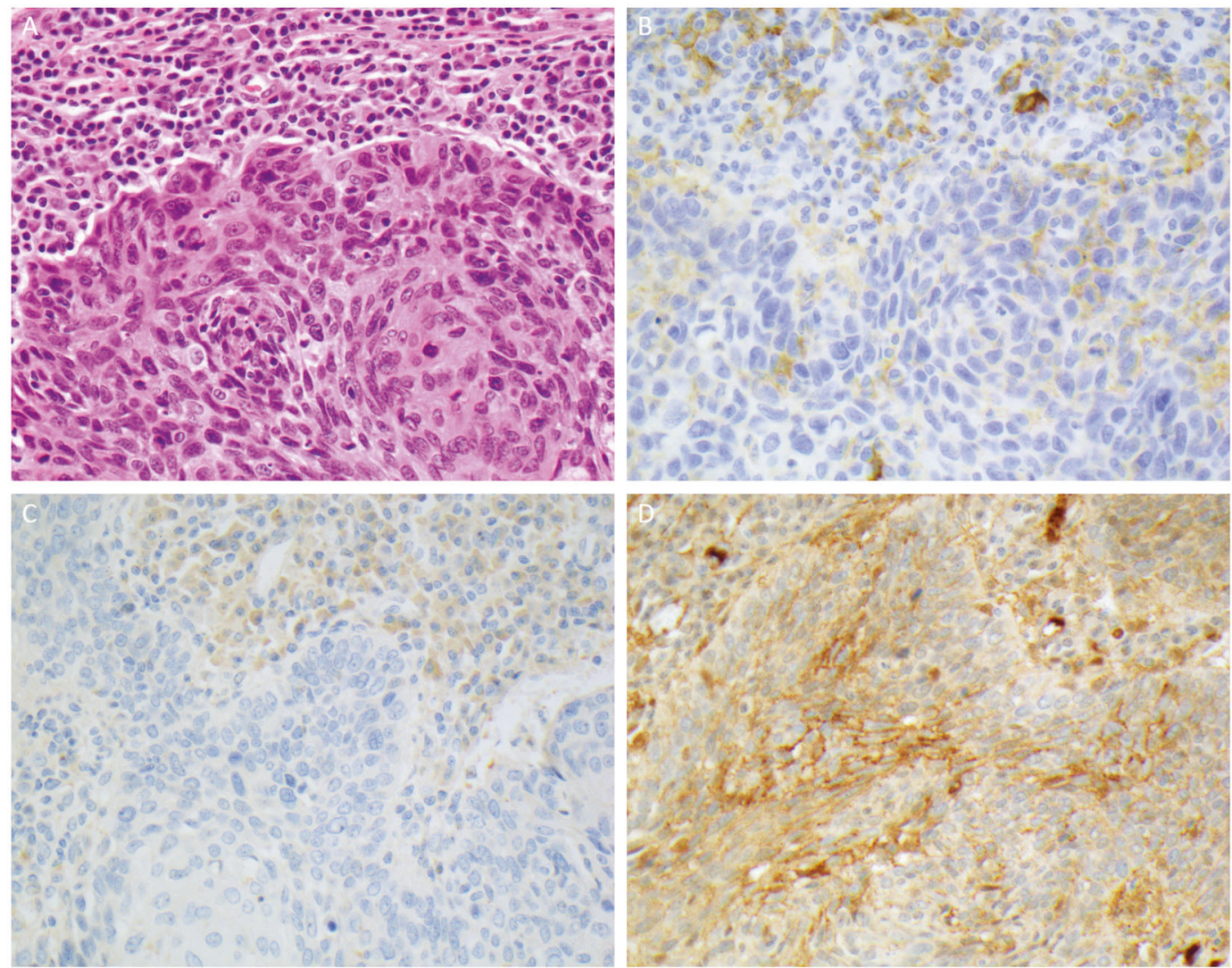

Fig. 3 PD-L1, TIM-3, and Gal-9 expression in vulvar squamous cell carcinoma. Like cervical squamous cell carcinomas, vulvar squamous cell carcinomas were usually PD-L1 positive in tumor cells and/or immune cells: this case (a) shows more prominent expression in peritumoral lymphocytes with scattered positivity in the tumor cells

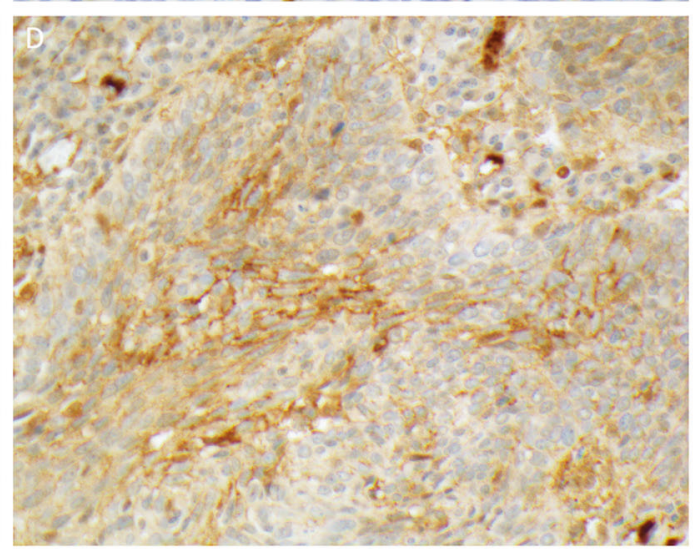

(b). TIM-3 expression as less impressive in this case, with staining limited to lymphocytes (c). Gal-9 (d) showed immune cell positivity as well as decreased nuclear expression and prominent membranous expression in tumor cells. 
Table 2 PD-L1 expression and co-expression with TIM-3 and GAL-9.

\begin{tabular}{|c|c|c|c|}
\hline & $\begin{array}{l}\text { Tumoral PD- } \\
\text { L1 } \geq 1 \%\end{array}$ & $\begin{array}{l}\text { PD-L1 } \\
\text { CPS } \geq 1\end{array}$ & $\begin{array}{l}\text { TIM-3, Gal-9, } \\
\& \text { PD-L1 Co- } \\
\text { expression } \\
(\mathrm{CPS} \geq 1)\end{array}$ \\
\hline $\begin{array}{l}\text { All Intraepithelial } \\
\text { Lesions }(\boldsymbol{n}=29)\end{array}$ & $\begin{array}{l}\mathbf{1 0 \%}(3 / 29) \\
\text { Staining } \\
\text { extent: } \\
1-5 \%: 3\end{array}$ & $\begin{array}{l}\text { 21\% }(6 / 29) \\
\text { CPS value: } \\
\text { 1-5: } 2 \\
\text { 11-25: } 1 \\
\text { 25-50: } 3\end{array}$ & $7 \%(2 / 29)$ \\
\hline CIN $3(n=14)$ & 0\% $(0 / 14)$ & 0\% $(0 / 14)$ & 0\% (0/14) \\
\hline $\operatorname{VIN} 3(n=13)$ & $\begin{array}{l}32 \%(3 / 13) \\
\text { Staining } \\
\text { extent: } \\
1-5 \%: 3\end{array}$ & $\begin{array}{l}\mathbf{3 8 \%}(5 / 13) \\
\text { CPS value: } \\
\text { 1-5: } 2 \\
\text { 11-25: } 1 \\
\text { 25:50: } 2\end{array}$ & $15 \%(2 / 13)$ \\
\hline$d V I N(n=2)$ & 0\% $(0 / 2)$ & $\begin{array}{l}\mathbf{5 0 \%}(1 / 2) \\
\text { CPS value: } \\
\text { 25-50: } 1\end{array}$ & $\mathbf{0 \%}(0 / 2)$ \\
\hline $\begin{array}{l}\text { All Invasive SCC } \\
(\boldsymbol{n}=34)\end{array}$ & $\begin{array}{l}71 \%(24 / 34) \\
\text { Staining } \\
\text { extent: } \\
1-5 \%: 9 \\
6-10 \%: 5 \\
11-25 \%: 4 \\
25-50 \%: 4 \\
>50 \%: 2\end{array}$ & $\begin{array}{l}\mathbf{8 2 \%} \\
(28 / 34) \text { CPS } \\
\text { Value: } \\
\text { 11-25: } 4 \\
\text { 25-50: } 11 \\
>50: 13\end{array}$ & $82 \%(28 / 34)$ \\
\hline $\begin{array}{l}\text { Cervical SCC } \\
(n=15)\end{array}$ & $\begin{array}{l}\mathbf{7 3 \%}(11 / 15) \\
\text { Staining } \\
\text { extent: } \\
1-5 \%: 3 \\
6-10 \%: 1 \\
11-25 \%: 3 \\
25-50 \%: 3 \\
>50 \%: 1\end{array}$ & $\begin{array}{l}\mathbf{8 7 \%} \\
(13 / 15) \text { CPS } \\
\text { value: } \\
\text { 11-25: } 2 \\
\text { 25:50: } 5 \\
>50: 6\end{array}$ & $\mathbf{8 7 \%}(13 / 15)$ \\
\hline $\begin{array}{l}\text { Vulvar } S C C, H P V- \\
\text { Associated }(n=16)\end{array}$ & $\begin{array}{l}\text { 63\% }(10 / 16) \\
\text { Staining } \\
\text { extent: } \\
1-5 \%: 5 \\
6-10 \%: 3 \\
11-25 \%: 1 \\
25-50 \%: 0 \\
>50 \%: 1\end{array}$ & $\begin{array}{l}\mathbf{8 1 \%} \\
(13 / 16) \text { CPS } \\
\text { value: } \\
\text { 11-25: } 2 \\
\text { 25-50: } 6 \\
>50: 5\end{array}$ & $\mathbf{8 1 \%}(13 / 16)$ \\
\hline $\begin{array}{l}\text { Vulvar SCC, } \\
\text { dVIN-associated } \\
(n=3)\end{array}$ & $\begin{array}{l}\mathbf{1 0 0 \%}(3 / 3) \\
\text { Staining } \\
\text { extent: } \\
1-5 \%: 1 \\
6-10 \%: 1 \\
11-25 \%: 0 \\
25-50 \%: 1\end{array}$ & $\begin{array}{l}67 \%(2 / 3) \\
\text { CPS value: } \\
>50: 2\end{array}$ & $67 \%(2 / 3)$ \\
\hline
\end{tabular}

The proportion of positive cases (for tumoral staining $\geq 1 \%$; for CSP $\geq$ 1 ) is indicated in bold. The number of PD-L1-positive cases falling into different expression ranges cascades beneath this value in individual tumoral and CPS columns. Co-expression with TIM-3 and Gal-9 is based on the CPS $\geq 1$ threshold for all three markers

squamous cell carcinomas (mean: 34\%, range: 5-70\%) (Fig. 5). A multivariate regression model showed that PD-L1 and TIM3 scores are significantly related to TIL stroma score ( $p=0.001$, Wilk's lambda statistic) (Fig. 6).

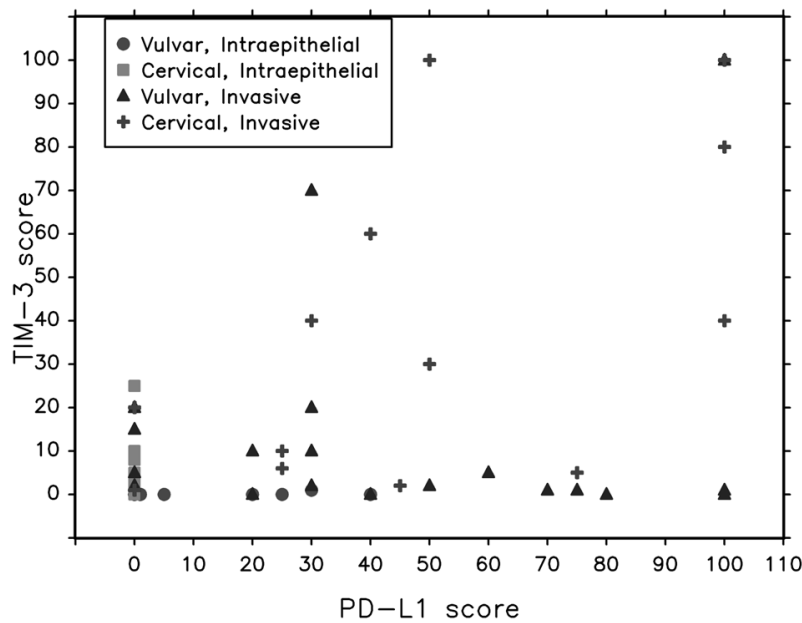

Fig. 4 Plot of TIM-3 and PD-L1 CPS scores. Notably, intraepithelial lesions are clustered at the lower left-hand side of the plot, reflecting low levels of expression for both checkpoint markers among noninvasive lesions, whereas squamous cell carcinomas of both cervical and vulvar origin demonstrate a range of expression patterns that includes high dual-expressers.

Table 3 TIM-3, Gal-9, and PD-L1 co-expression using the CPS.

\begin{tabular}{|c|c|c|}
\hline & $\begin{array}{l}\text { TIM-3 + \& } \\
\text { Gal-9 }+ \text { CPS } \geq 1\end{array}$ & $\begin{array}{l}\text { TIM-3+, Gal-9+, } \\
\& \text { PD-L } 1+\text { CPS } \geq 1\end{array}$ \\
\hline $\begin{array}{l}\text { All Intraepithelial Lesions } \\
(n=29)\end{array}$ & $41 \%(12 / 29)$ & $7 \%(2 / 29)$ \\
\hline CIN $3(n=14)$ & $64 \%(9 / 14)$ & 0\% $(0 / 14)$ \\
\hline VIN $3(n=13)$ & $23 \%(3 / 13)$ & $15 \%(2 / 13)$ \\
\hline $\mathrm{dVIN}(n=2)$ & $\mathbf{0 \%}(0 / 2)$ & $\mathbf{0 \%}(0 / 2)$ \\
\hline All Invasive SCC $(n=34)$ & $97 \%(33 / 34)$ & $82 \%(28 / 34)$ \\
\hline Cervical SCC $(n=15)$ & $100 \%(15 / 15)$ & $\mathbf{8 7 \%}(13 / 15)$ \\
\hline $\begin{array}{l}\text { Vulvar SCC, HPV- } \\
\text { associated }(n=16)\end{array}$ & $100 \%(16 / 16)$ & $\mathbf{8 1 \%}(13 / 16)$ \\
\hline $\begin{array}{l}\text { Vulvar SCC, dVIN- } \\
\text { associated }(n=3)\end{array}$ & $100 \%(3 / 3)$ & $67 \%(2 / 3)$ \\
\hline
\end{tabular}

\section{Discussion}

The checkpoint molecule TIM-3 is currently under investigation as an immunotherapeutic target in a variety of cancer types, including cervical and vulvar squamous cell carcinomas [8, 27-29]. We herein investigate expression of TIM-3 and its ligand, Gal-9, in cervical and vulvar neoplasia to ascertain the potential biologic basis for a possible clinical response to such therapies. We found that TIM-3 expression was significantly more common in invasive vs. intraepithelial lesions and was identified on both tumor cells and associated inflammatory cells. Its ligand, Gal-9, was nearly ubiquitous in immune cells and showed membranous expression in a subset of invasive and intraepithelial neoplasms. For invasive carcinomas, there was no significant 
difference in membranous TIM-3 or Gal-9 expression based on cervical vs. vulvar location; however, TIM-3 expression was significantly higher among CIN 3 when compared with VIN 3. There was no significant difference in expression based on HPV status, although statistical power was restricted by low numbers of HPV-negative cases. PD-L1 expression showed a similar pattern, with significantly higher expression among invasive vs. intraepithelial lesions and no significant differences in expression based on either cervical vs. vulvar localization or HPV association. Importantly, all PD-L1-positive cancers co-expressed both TIM-3 and Gal-9, suggesting that most cervical and vulvar squamous cell carcinomas enlist multiple mechanisms of

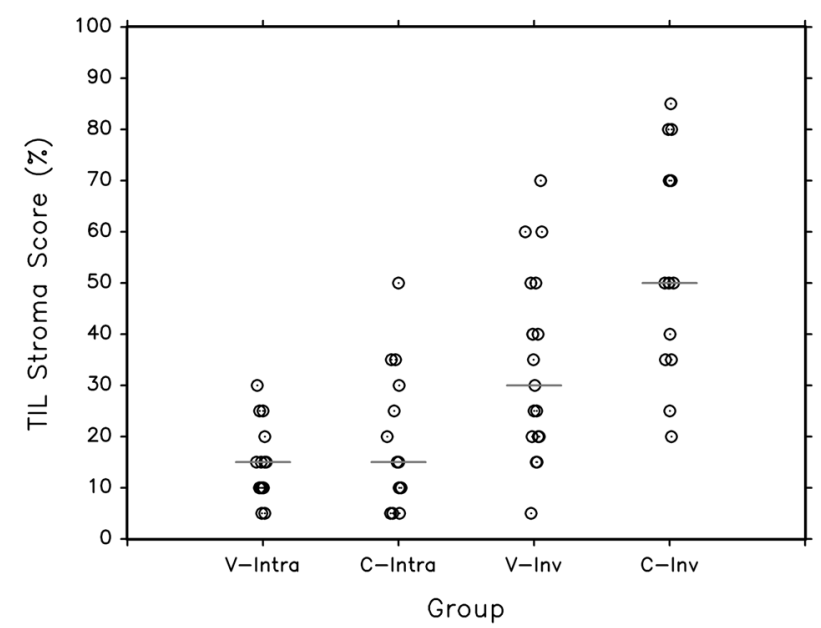

Fig. 5 Plot of TIL stroma score by group. The red lines are group medians. $P<0.001$, nonparametric Kruskal-Wallis test. Invasive squamous cell carcinomas had significantly more inflammation than intraepithelial lesions, with cervical carcinomas showing higher levels than vulvar carcinomas.
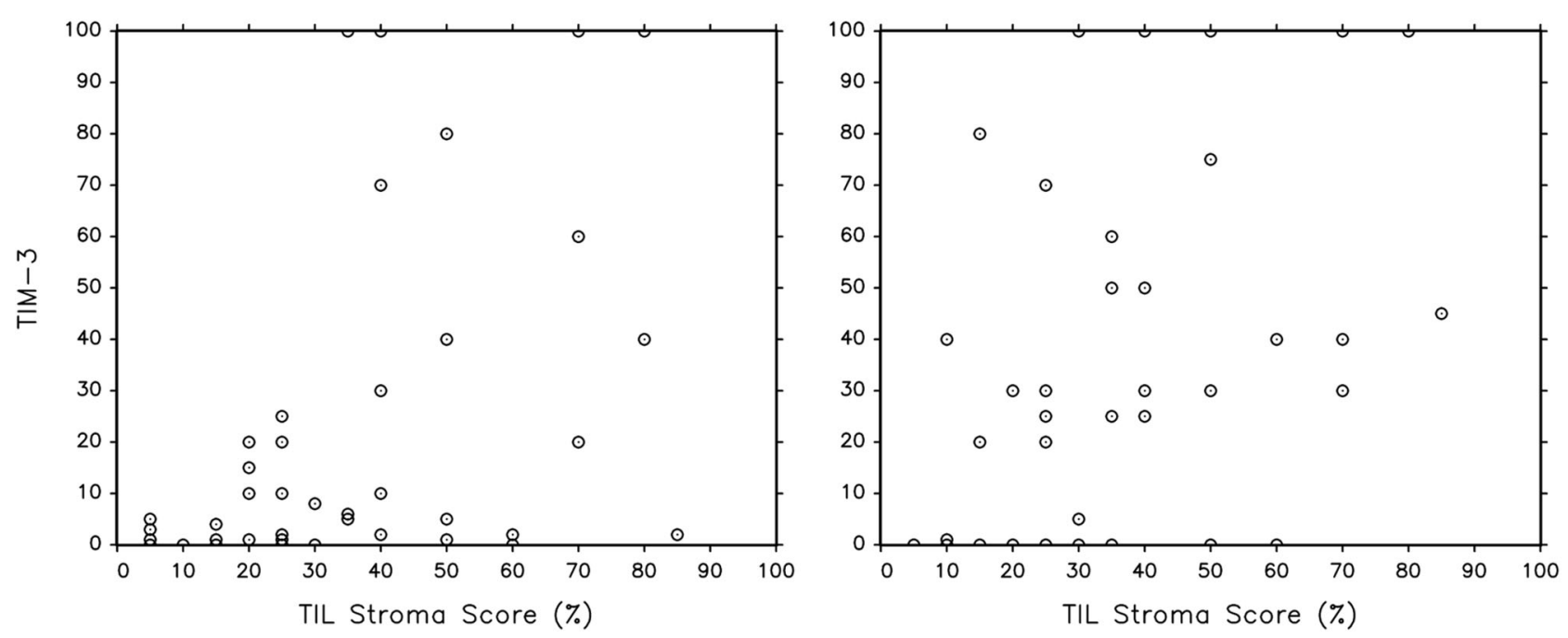

Fig. 6 Plot of TIL stromal score vs. PD-L1 and TIM-3. On multivariate regression analysis, PD-L1 and TIM3 scores were significantly related to TIL stroma Score ( $p=0.001$, Wilk's lambda statistic), after adjusting for site (cervical vs. vulvar) and lesion type (intraepithelial vs. invasive). checkpoint-based immunoevasion. Expression of both TIM3 and PD-L1 was significantly associated with TIL, with higher levels of checkpoint molecule expression seen in the context of increased inflammation in tumor-associated stroma.

The expression patterns of TIM-3 and Gal-9 provoke interesting questions. When drawing parallels to the PD-1/ PD-L1 immunosuppressive checkpoint axis, TIM-3 is thought to be analogous to PD-1 while its ligand, Gal-9, is presumably more analogous to PD-L1. As such, one might expect to see TIM-3 expression restricted to the lymphoid compartment, as is seen with PD-1. Unlike PD-1, however, TIM-3 was commonly seen not only on immune cells, but also on the cell surface in many invasive tumors. Although prior investigations on TIM-3 in gastric carcinoma, renal cell carcinoma, and melanoma have focused chiefly on its expression in tumor-associated immune cells, expression on tumor cells has also been identified in endometrial carcinoma $[15,16,30,31]$. While the immunotherapeutic significance of TIM-3 expression on tumor cells remains unclear, its frequent expression on lymphocytes in cervical and vulvar neoplasia suggests that TIM-3 immunosuppressive checkpoint axis is likely to be activated in these tumors, particularly given that its ligand, Gal-9, is frequently present on the tumor cell surface. The most clinically relevant scoring system for TIM-3 remains unknown, and clinical trials investigating the predictive significance of tumoral vs. tumor-associated immune cell expression would be valuable.

The staining patterns observed with Gal-9 also prompt questions. Gal-9 shared PD-L1's propensity for both membranous epithelial and immune cell expression in tumors, but also showed strong nuclear and variable 
cytoplasmic expression in both neoplastic lesions and, more prominently, in adjacent benign tissues. Interestingly, nuclear/cytoplasmic staining was often diminished in the context of membranous staining. The widespread distribution of Gal-9 likely derives from the range of activity attributed to galectins. In addition to its role as an immunomodulatory ligand, Gal-9 plays a role in cell adhesion, with loss of expression tied to increased metastatic capability and decreased differentiation in some cancers [15, 32-34]. As with TIM-3, the most appropriate scoring system for Gal-9 remains unclear, and investigations assessing response to checkpoint inhibition with different patterns of tumoral expression as well as immune cell expression would be interesting.

The relationships between TIM-3, Gal-9, and PD-L1 have been investigated only recently. Sideras et al. evaluated Gal-9 and PD-L1 expression in hepatocellular carcinoma and found that the combination of PD-L1 expression with loss of nuclear/cytoplasmic Gal-9 staining imparted worsened prognosis when compared with either finding in isolation [35]. This suggests that the immunosuppressive properties of Gal-9 may be significant for cancer progression. However, the study did not assess TIM-3 expression, nor did it specifically evaluate whether decreased nuclear/ cytoplasmic Gal-9 expression corresponded with increased Gal-9 membranous staining, as was observed in our study. There is also limited data on TIM-3 and PD-L1 coexpression. Several studies have revealed a significant increase in PD-1 and TIM-3-positive lymphocytes in association with breast carcinomas, hepatocellular carcinomas, and squamous cell carcinomas [36-38], but dual expression of TIM-3 and PD-L1 has not been well-studied.

The fact that PD-L1 expression was often accompanied by TIM-3/Gal-9 staining in our series highlights the extreme complexity of the immunologic milieu of cervical and vulvar neoplasms and suggests that most of these tumors enlist multiple modes of immune evasion. Notably, expression of both targetable checkpoints is significantly elevated in the context of increased inflammation, supporting the thesis that these mechanisms represent an adaptive response to increased anti-tumoral activity by the host immune system. In the current study, all of PD-L1positive invasive carcinomas showed some level of tumoral and/or immune TIM-3 and Gal-9 co-expression, suggesting that both mechanisms of immune evasion may be at play in these neoplasms. This may help account for the limited responses of many cervical cancers to monotherapy targeting the PD-1/PD-L1 axis [4, 7]. In contrast, anti-TIM-3 checkpoint inhibition may have independent value in the subset of squamous cell carcinomas, which are TIM-3/ GAL-3-positive but PD-L1-negative.

Although no relationship was found between TIM-3, Gal9, or PD-L1 in HPV-associated vs. HPV-unassociated vulvar intraepithelial lesions and cancers in this study, this represents an area for possible future inquiry as the current case set included only a few HPV-negative cases. That said, existing studies have failed to demonstrate a significant relationship between HPV status and PD-L1 expression in vulvar tumors, therefore despite the thesis that a virally-driven malignancy may prove more immunogenic, immunoevasion may be no more critical for carcinogenesis in that setting than in tumors which are unaffiliated with a viral etiology [39, 40].

Another area for future study is the possible role of topical immunotherapy in squamous intraepithelial lesions of the cervix and vulva. Although the low levels of checkpoint molecule expression observed in these lesions argues against a role for systemic immunotherapy in this setting, direct application may be relevant should such localized treatment options become available. The toll-like receptor agonist Imiquimod has long been used to treat cervical and vulvar dysplasias with some success [41-43], suggesting that this method of therapy could have promise in pre-invasive neoplasia in these sites. It will be of interest to see whether anti-PD-1/PD-L1 and/or anti-TIM-3 treatments have a role in this context.

In summary, the targetable immunosuppressive checkpoint molecule TIM-3 and its ligand, Gal-9, were highly expressed in cervical and vulvar squamous cell carcinomas and show only limited expression in intraepithelial lesions. All PD-L1-positive squamous cell carcinomas co-expressed these markers, suggesting that combination approaches to checkpoint inhibition may have value in treating these neoplasms. Higher levels of both PD-L1 and TIM-3 were significantly associated with increased tumor-associated inflammation, suggesting that checkpoint inhibition may be most successful in highly inflamed tumors. These findings could inform future clinical trials investigating combination immunotherapeutic approaches to cervical and vulvar squamous cancer.

Acknowledgements The authors would like to thank the University of Virginia Biorepository and Tissue Research Facility for their skill and expertise in performing all immunohistochemical staining.

\section{Compliance with ethical standards}

Conflict of interest The authors declare that they have no conflict of interest.

Publisher's note Springer Nature remains neutral with regard to jurisdictional claims in published maps and institutional affiliations.

\section{References}

1. Topalian SL, Hodi FS, Brahmer JR, Gettinger SN, Smith DC, McDermott DF, et al. Safety, activity, and immune correlates of anti-PD-1 antibody in cancer. N Engl J Med. 2012;366: 2443-54. 
2. Gadducci A, Guerrieri ME. Immune checkpoint inhibitors in gynecological cancers: update of literature and perspectives of clinical research. Anticancer Res. 2017;37:5955-65.

3. Taube JM, Klein A, Brahmer JR, Xu H, Pan X, Kim JH, et al. Association of PD-1, PD-1 ligands, and other features of the tumor immune microenvironment with response to anti-PD-1 therapy. Clin Cancer Res. 2014;20:5064-74.

4. Frenel JS, Le Tourneau C, O’Neil B, Ott PA, Piha-Paul SA, Gomez-Roca C, et al. Safety and efficacy of pembrolizumab in advanced, programmed death ligand 1-positive cervical cancer: results from the phase Ib KEYNOTE-028 Trial. J Clin Oncol. 2017;35:4035-41.

5. Chung HC, Ros W, Delord JP, Perets R, Italiano A, ShapiraFrommer R, et al. Efficacy and safety of pembrolizumab in previously treated advanced cervical cancer: results from the phase II KEYNOTE-158 study. J Clin Oncol. 2019;37:1470-8.

6. Shields LBE, Gordinier ME. Pembrolizumab in recurrent squamous cell carcinoma of the vulva: case report and review of the literature. Gynecol Obstet Invest. 2019;84:94-8.

7. Frenel JS, Le Tourneau C, O'Neil BH, Ott PA, Piha-Paul SA, Gomez-Roca CA, et al. Pembrolizumab in patients with advanced cervical squamous cell cancer: preliminary results from the phase Ib KEYNOTE-028 study. J Clin Oncol. 2016;34.

8. Dempke WCM, Fenchel K, Uciechowski P, Dale SP. Second- and third-generation drugs for immuno-oncology treatment-The more the better? Eur J Cancer. 2017;74:55-72.

9. Vanderstraeten A, Luyten C, Verbist G, Tuyaerts S, Amant F. Mapping the immunosuppressive environment in uterine tumors: implications for immunotherapy. Cancer Immunol Immunother. 2014;63:545-57.

10. Zhu C, Anderson AC, Schubart A, Xiong H, Imitola J, Khoury SJ, et al. The Tim-3 ligand galectin- 9 negatively regulates $T$ helper type 1 immunity. Nat Immunol. 2005;6:1245-52.

11. Sánchez-Fueyo A, Tian J, Picarella D, Domenig C, Zheng XX, Sabatos CA, et al. Tim-3 inhibits T helper type 1-mediated auto- and alloimmune responses and promotes immunological tolerance. Nat Immunol. 2003;4:1093-101.

12. Das M, Zhu C, Kuchroo VK. Tim-3 and its role in regulating antitumor immunity. Immunol Rev. 2017;276:97-111.

13. Cao Y, Zhou X, Huang X, Li Q, Gao L, Jiang L, et al. Tim-3 expression in cervical cancer promotes tumor metastasis. PLoS ONE. 2013;8:e53834.

14. Zhu D, Jiang XH, Jiang YH, Ding WC, Zhang CL, Shen H, et al. Amplification and overexpression of TP63 and MYC as biomarkers for transition of cervical intraepithelial neoplasia to cervical cancer. Int J Gynecol Cancer. 2014;24:643-8.

15. Jiang J, Jin M-S, Kong F, Cao D, Ma HX, Jia Z, et al. Decreased galectin-9 and increased Tim-3 expression are related to poor prognosis in gastric cancer. PLoS ONE. 2013;8:e81799.

16. Moore M, Ring KL, Mills AM. TIM-3 in endometrial carcinomas: an immunotherapeutic target expressed by mismatch repairdeficient and intact cancers. Mod Pathol. 2019;32:1168-79.

17. Zhou X, Sun L, Jing D, Xu G, Zhang J, Lin L, et al. Galectin-9 expression predicts favorable clinical outcome in solid tumors: a systematic review and meta-analysis. Front Physiol. 2018;9:452.

18. Choi SIl, Seo Kwoo, Kook MC, Kim CG, Kim YW, Cho SJ, et al. Prognostic value of tumoral expression of galectin-9 in gastric cancer. Turkish J Gastroenterol. 2017;28:166-70.

19. Zhang Z-Y, Dong J-H, Chen Y-W, Wang XQ, Li CH, Wang J, et al. Galectin-9 acts as a prognostic factor with antimetastatic potential in hepatocellular carcinoma. Asian Pac J Cancer Prev. 2012;13:2503-9.

20. Yoon HK, Kim TH, Park S, Jung H, Quan X, Park SJ, et al. Effect of anthracycline and taxane on the expression of programmed cell death ligand-1 and galectin-9 in triple-negative breast cancer. Pathol-Res Pract. 2018;214:1626-31.
21. Nobumoto A, Nagahara K, Oomizu S, Katoh S, Nishi N, Takeshita K, et al. Galectin-9 suppresses tumor metastasis by blocking adhesion to endothelium and extracellular matrices. Glycobiology. 2008; 18:735-44.

22. Xu H, Lin G, Huang C, Zhu W, Miao Q, Fan X, et al. Assessment of concordance between $22 \mathrm{C} 3$ and SP142 immunohistochemistry assays regarding PD-L1 expression in non-small cell lung cancer. Sci Rep. 2017;7:16956.

23. Rimm DL, Han G, Taube JM, Yi ES, Bridge JA, Flieder DB, et al. A prospective, multi-institutional, pathologist-based assessment of 4 immunohistochemistry assays for PD-L1 expression in non-small cell lung cancer. JAMA Oncol. 2017;3:1051-8.

24. Kulangara K, Zhang N, Corigliano E, Guerrero L, Waldroup $\mathrm{S}$, Jaiswal D, et al. Clinical utility of the combined positive score for programmed death ligand-1 expression and the approval of pembrolizumab for treatment of gastric cancer. Arch Pathol Lab Med. 2018;143:330-7.

25. Salgado R, Denkert C, Demaria S, Sirtaine N, Klauschen $F$, Pruneri $G$, et al. The evaluation of tumor-infiltrating lymphocytes (TILS) in breast cancer: Recommendations by an International TILS Working Group 2014. Ann Oncol. 2015; 26:259-71.

26. Mills AM, Zadeh S, Sloan EA, Chinn Z, Modesitt SC, Ring KL, et al. Indoleamine 2,3-dioxygenase in endometrial cancer: a targetable mechanism of immune resistance in mismatch repairdeficient and intact endometrial carcinomas. Mod Pathol. 2018; 31:1282-90.

27. Li X, Hu W, Zheng $\mathrm{X}$, Zhang $\mathrm{C}$, Du P, Zheng Z, et al. Emerging immune checkpoints for cancer therapy. Acta Oncol. 2015;54:1706-13.

28. Du W, Yang M, Turner A, Xu C, Ferris RL, Huang J, et al. TIM3 as a target for cancer immunotherapy and mechanisms of action. Int J Mol Sci. 2017;18:10.

29. Davar D, Boasberg PD, Eroglu Z, et al. A phase 1 study of TSR022, an anti-TIM-3 monoclonal antibody, in combination with TSR-042 (anti-PD-1) in patients with colorectal cancer and postPD-1 NSCLC and melanoma. J Immunother Cancer. 2018;6 Suppl 1:115.

30. Fourcade J, Sun Z, Benallaoua M, Guillaume P, Luescher IF, Sander C, et al. Upregulation of Tim-3 and PD-1 expression is associated with tumor antigen-specific CD8 $+\mathrm{T}$ cell dysfunction in melanoma patients. J Exp Med. 2010;207:2175-86.

31. Fourcade J, Sun Z, Pagliano O, Chauvin JM, Sander C, Janjic B, et al. PD-1 and Tim-3 regulate the expansion of tumor antigenspecific CD8(+) T cells induced by melanoma vaccines. Cancer Res. 2014;74:1045-55.

32. Liang M, Ueno M, Oomizu S, Arikawa T, Shinonaga R, Zhang $S$, et al. Galectin-9 expression links to malignant potential of cervical squamous cell carcinoma. J Cancer Res Clin Oncol. 2008;134:899-907.

33. Irie A, Yamauchi A, Kontani K, Kihara M, Liu D, Shirato Y, et al. Galectin-9 as a prognostic factor with antimetastatic potential in breast cancer. Clin Cancer Res. 2005;11:2962-8.

34. Kageshita T, Kashio Y, Yamauchi A, Seki M, Abedin MJ, Nishi Net, al. Possible role of galectin-9 in cell aggregation and apoptosis of human melanoma cell lines and its clinical significance. Int J Cancer. 2002;99:809-16.

35. Sideras K, Biermann K, Verheij J, Takkenberg BR, Mancham S, Hansen BE, et al. PD-L1, Galectin-9 and CD8+ tumor-infiltrating lymphocytes are associated with survival in hepatocellular carcinoma. Oncoimmunology. 2017;6:e1273309.

36. Sasidharan Nair V, El Salhat H, Taha RZ, John A, Ali BR, Elkord Eet. DNA methylation and repressive H3K9 and H3K27 trimethylation in the promoter regions of PD-1, CTLA-4, TIM-3, LAG-3, TIGIT, and PD-L1 genes in human primary breast cancer. Clin Epigenetics. 2018;10:78. 
37. Li Z, Li N, Li F, Zhou Z, Sang J, Chen Y, et al. Immune checkpoint proteins PD-1 and TIM-3 are both highly expressed in liver tissues and correlate with their gene polymorphisms in patients with HBVrelated hepatocellular carcinoma. Med (Baltim). 2016;95:e5749.

38. Linedale R, Schmidt C, King BT, Ganko AG, Simpson F, Panizza BJ, et al. Elevated frequencies of CD8 T cells expressing PD-1, CTLA-4 and Tim-3 within tumour from perineural squamous cell carcinoma patients. PLoS ONE. 2017;12:e0175755.

39. Thangarajah F, Morgenstern B, Pahmeyer C, Schiffmann LM, Puppe J, Mallmann P, et al. Clinical impact of PD-L1 and PD-1 expression in squamous cell cancer of the vulva. J Cancer Res Clin Oncol. 2019;145:1651-60.

40. Choschzick M, Gut A, Fink D. PD-L1 receptor expression in vulvar carcinomas is HPV-independent. Virchows Arch. 2018;473:513-6.
41. van de Sande AJM, Koeneman MM, Gerestein CG, Kruse AJ, van Kemenade FJ, van Beekhuizen HJ. TOPical Imiquimod treatment of high-grade Cervical intraepithelial neoplasia (TOPIC trial): study protocol for a randomized controlled trial. BMC Cancer. 2018;18:655.

42. Grimm C, Polterauer S, Natter C, Rahhal J, Hefler L, Tempfer CB, et al. Treatment of cervical intraepithelial neoplasia with topical imiquimod: a randomized controlled trial. Obstet Gynecol. 2012; 120:152-9.

43. Hurt CN, Jones SEF, Madden TA, Fiander A, Nordin AJ, Naik R, et al. Recurrence of vulval intraepithelial neoplasia following treatment with cidofovir or imiquimod: results from a multicentre, randomised, phase II trial (RT3VIN). BJOG Int J Obstet Gynaecol. 2018;125:1171. 